\title{
Assessment of the Till-U-Test GC slide
}

\author{
W H DONALD \\ From the Department of Genitourinary Medicine, Derbyshire Royal Infirmary, Derby
}

SUMMARY The Till-U-Test (TUT) GC slide, a simple cultural procedure for Neisseria gonorrhoeae, was compared with a standard procedure using modified Thayer-Martin medium for laboratory confirmation of gonorrhoea in 144 patients. The TUT proved to be slightly more sensitive than the standard procedure. In $92 \cdot 6 \%$ of all patients the results agreed, and in cases with positive results to Gram stain $N$ gonorrhoeae was isolated in $93.6 \%$ with the TUT compared with $\mathbf{8 8 . 9 \%}$ with the standard procedure. The TUT slide was easy to handle and produced results sooner than conventional methods. Thus the TUT could be very useful for presumptive diagnosis of gonorrhoea when full laboratory facilities are not readily available, as very little equipment is required.

\section{Introduction}

Numerous methods for laboratory diagnosis of gonorrhoea are available, including simplified tests such as the Microcult GC. ${ }^{1}$ This study was undertaken to assess the recently developed cultural procedure, the Till-U-Test (TUT) GC slide (Tillomed Laboratories), for the growth of Neisseria gonorrhoeae from a direct inoculum on a ThayerMartin medium with colistin replacing polymixin. Transport media are unnecessary, as the inoculation can be made with a swab or loop of material from the patient and the slide placed immediately in its own $\mathrm{CO}_{2}$ environment. The inoculated slide may then be incubated in the clinic or sent to the laboratory for incubation and full identification. The slide consists of a sterile tongue covered on both sides with a brown medium. The tongue is fitted to a cap which screws into a sterile container, inside which a $\mathrm{CO}_{2}$ atmosphere can be generated. The TUT was assessed in comparison with an established selective cultural method for its ability to detect $N$ gonorrhoeae in clinical specimens containing the organism.

\section{Patients and methods}

The TUT was used in 67 male patients being investigated for urethritis and in 77 female patients presenting with genitourinary symptoms or as contacts of a male patient with gonorrhoea or nonAddress for reprints: Dr W H Donald, Department of Genitourinary
Medicine, Derbyshire Royal Infirmary, Derby

Received for publication 11 August 1979 specific urethritis. A total of 221 specimens were examined. In all cases Gram-stained smears were examined for Gram-negative intracellular diplococci.

Specimens for Gram-staining were taken from each patient with a sterile platinum loop. Specimens for culture were taken with a sterile swab; after inoculation of the TUT the swab was placed in Amies's transport medium. The TUT was inoculated according to the manufacturers' instructions by rubbing the swab gently over the surface of both sides of the slide; before the slide was replaced in the container the contents of a $\mathrm{CO}_{2}$ generating capsule were tipped on to a moist sponge at the bottom of the container and the tongue replaced. The date and time of inoculation were entered on the label. The container was then transferred immediately to an incubator. The TUT container and the swab in Amies's transport medium were both taken to the microbiological laboratory between two and 18 hours after inoculation.

In the laboratory incubation of the TUT continued at $37^{\circ} \mathrm{C}$. The swab was inoculated on to a nonselective medium (ordinary blood agar plate) and on to a selective medium, Thayer-Martin medium with the addition of trimethoprim and a reduction in concentration of vancomycin $(1500 \mu \mathrm{g} / \mathrm{ml})$ and polymixin $(20000 \mathrm{IU} / \mathrm{ml})$. Cultures were examined 48 hours after inoculation and suspect colonies tested with oxidase reagent. Oxidase-positive colonies were subcultured for fermentation reactions and a specimen from the colony was Gram-stained to confirm the presence of Gram-negative diplococci. $N$ gonorrhoeae were identified as Gram-negative diplococci with typical-looking oxidase-positive colonies which fermented glucose only. 


\section{Results}

There was a close correlation between the findings of the two cultural methods; slightly more positive results were obtained with the TUT than with the standard cultural procedure (Table I). In 133 $(92 \cdot 3 \%)$ of the 144 patients the results agreed, and, of the 63 cases with typical-looking organisms on Gram-stain, positive results were obtained with the TUT in $59(93.6 \%)$ compared with $56(88.9 \%)$ with standard cultures. Of the 81 cases showing no organisms on Gram-stained smear, positive results were obtained with the TUT in three $(3 \cdot 7 \%)$ cases and with standard cultures in two $(2 \cdot 5 \%)$ (Table II). The detection rate for $N$ gonorrhoeae from all specimens was high, particularly from cervical swabs (Table II).

TABLE I Results of Till-U-Test compared with those using standard cultures from 144 patients

\begin{tabular}{llllllllllll}
\hline Method & Results \\
\hline Gram-stain & + & + & + & + & - & - & - & - \\
TUT & + & + & - & - & + & + & - & - \\
Standard culture & + & - & + & - & + & - & + & - \\
No of patients & 53 & 6 & 3 & 1 & 2 & 1 & 0 & 78 \\
\hline & Positive for $N$ \\
\hline
\end{tabular}

TABLE II Isolation of $N$ gonorrhoeae from total specimens

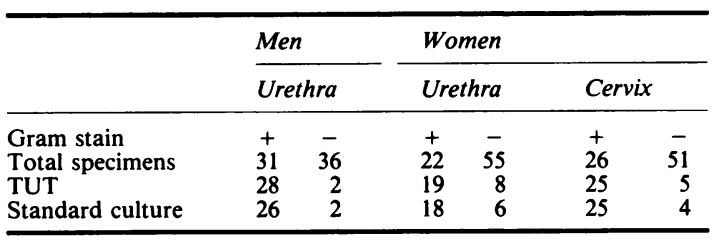

+ Positive for $N$ gonorrhoeae - negative

\section{Discussion}

A Gram stain of urethral discharge in men has been regarded as $99 \%$ accurate in the diagnosis of gonorrhoea if positive results are obtained, ${ }^{2}$ but gonococci may be recovered by culture in $>5 \%$ of cases with negative results ${ }^{3}$ which were diagnosed initially as non-specific urethritis. Cultural diagnosis is therefore desirable in cases with negative results to Gram stain. In women, Gram stains are much less reliable, and cultures are therefore essential for a satisfactory diagnosis.

The principle of the TUT is a presumptive diagnosis of gonorrhoea by means of growth on a selective medium of typical colonial characteristics, oxidase reaction, and Gram stain. The test would not distinguish other Neisseria so it could not be reliably used for pharyngeal specimens, but when such facilities were available subculturing for sugar fermentation for further identification could be carried out.

The TUT stores satisfactorily for a minimum of three months at room temperature and requires only sterile swabs, Gram-staining equipment, a microscope, and an incubator. As growth was often evident after 24 hours' incubation, a combination of TUT and immunofluorescent staining could provide a report within 24 to 36 hours.

A great advantage of the TUT is that it can be incubated directly in its own $\mathrm{CO}_{2}$ atmosphere, so that transfer problems associated with conventional methods do not arise. The TUT could be a valuable diagnostic aid where full laboratory facilities are not immediately available.

I thank Mrs Kathryn Boddy, who supervised and reported on the Till-U-Test GC slides in the Microbiology Laboratory, and Mr L Glick of Tillomed Laboratories Ltd for kindly supplying the Till-U-Test GC slides.

\section{References}

1. Willcox RR, John J. Simplified method for the cultural diagnosis of gonorrhoea. $\mathrm{Br} J$ Vener Dis 1976; 52: 256.

2. Fiumara NS. The diagnosis and treatment of gonorrhoea. Med Clin North Am 1972; 56: 1105-14.

3. Willcox RR, Sparrow RW, Fazluddin GM. Minocycline in the treatment of non-gonococcal urethritis. Br J Vener Dis 1975; 51: 206-9. 